\title{
The Role of Memory in Consecutive Translation
}

\author{
Khrystyna Pavliuk 1 *[0000-0002-1459-1628], Polina Melnyk 1 [0000-0001-6657-9498], \\ Viktoriia Antufieva 2 [0000-0001-9056-5475] \\ ${ }^{1}$ Odesa Polytechnic State University, Ukraine \\ ${ }^{2}$ Odesa State University of Internal Affairs, Ukraine \\ *khrystia0906@gmail.com
}

\begin{abstract}
In modern conditions, translation activity is associated with the semantic processing of information, the value of which is determined by socio-economic and legal categories, and translators are considered as subjects that ensure national security in the information sphere. A translator today is a person capable of handling huge flows of information in his professional activity. The paper focuses on the psychological aspects of translation activity, special attention is paid to memory as the main mechanism of speech activity. Development of memory flexibility, i.e. the ability to structure material, to group, creating semantic blocks, helps to retain large amounts of information in memory. The ability to organize the material is also necessary for effective translation activities.
\end{abstract}

Keywords: translation, consecutive interpretation, memory, psychological mechanism.

\section{INTRODUCTION}

Translation and interpretation in the work of a translator are carried out as a single process $[1,2]$. In science, interpretation is investigated from the point of view of the characteristics of human consciousness, which functions through the perception and processing of information. A feature of oral translation is the combination of various kinds of actions: listening to information, processing it and generating a message into the target language, which presupposes the active functioning of the psychological mechanisms of memory, attention, perception and thinking. Memory is one of the important mechanisms of translation activity.

The analysis of the translator's activity leads us to the traditional topics of psychology, which include states, properties, and features of cognitive processes.

\section{PURPOSE AND METHODS OF RESEARCH}

The purpose of the paper is to estimate the effectiveness of mechanism for supporting the interpretation process in order to improve the organization of training of future translators and interpreters.

To achieve the purpose, a number of investigation tasks are to be considered, such as:
1) to clarify the specifics of oral translation activities,

2) to examine format of interpretation as a complex of reproductive-receptive type of speech activity;

3) to monitor students' attitudes towards difficulties they have during simultaneous interpretation

Methodologically, the present analysis is based on the data obtained through a survey of challenges and impact of psychological mechanisms on the process of interpretation, in which 30 respondents expressed their attitude.

The main methods of the present paper are empirical and interpretive. The empirical methods consisted in conducting the survey of masters, graduate students (practicing translators) of the specialty "Philology" and "Translation Studies", their opinions about difficulties they have during simultaneous interpretation. The interpretive methods included the comprehensive and systematic study of the key problem connected with specifics of oral translation activities from a psychological point of view.

The results of the survey are analyzed below. 


\section{THEORETICAL BACKGROUND OF RESEARCH}

According to the definition formulated by V. N. Komissarov [3], interpretation is a type of translation in which the original and its translation appear in a non-fixed form, which predetermines the one-time perception by the translator of original segments and impossibility of subsequent comparison or correction of translation after its completion. Thus, memory is the most important mechanism for supporting the interpretation process. At the same time, according to the classification of V.I. Ermolovich, memory refers to general psychological mechanisms that provide the process of oral translation along with perception and comprehension [4, p. 50].

It is generally accepted that thinking begins with a specific task, requiring personal emotional involvement. Completion of mental activity is associated with finding an acceptable solution, achieving the goal. At the same time, the thought process is a volitional action that requires perseverance and constant focus on the desired result [5]. According to the figurative expression of N.S. Mavlevich, who is the translator of numerous French literature creations, "translation is thinking in pictures" [6]. This emotional-sensory perception is by no means devoid of theoretical basis.

There is one type at the center of our research interest - oral consecutive interpretation (hereinafter - OCI), by which we mean audible, direct, one-sided, contact, not requiring equipment translation, used in situations of conference interpretation, involving recording in the process of perceiving the source messages and the generation of a translation based on these records after the end of a large fragment of speech.

Various types of translation differ from each other in the set and sequence of actions, operations and methods of performing this activity. In the OCI, the chain of actions unfolds as follows: the translator perceives a fragment of the original message by ear, makes notes at the moment of its pronunciation, and, based on the records, generates a message in the target language.

Thoughts in a translator's head follow completely different paths during different translations - oral or written, simultaneous or sequential. It can be assumed that all our work can be reduced to what is usually denoted by the sociolinguistic term "code switching". However, in a person's head, communication code changes (that is, he/she switches the codes) when he/she has to change his/her social circle during the day and, as a result, move from one socio-cultural code to another [7]. This happens, for example, when we first communicate with a consultant in a store or a taxi driver, and then meet in the office with the head of organization. But in general, this term has not been used for a long time and the theory of "codes" is gradually becoming a thing of the past.

Without knowing an accurate translation, an experienced translator will never say this, he will try to find a synonym or convey the author's thought descriptively.

1. We hear or see unfamiliar text in a foreign language. Almost at the same moment, a possible translation is already being formed in my thoughts. If you synchronize, then you simply don't have time for doubts, because the speaker continues to speak. Try to polish your translation - and you won't translate anything else, just skip what has been said.

2. Experienced simultaneous interpreters note that at some point, simultaneously with listening and speaking, a parallel process of prediction develops in the interpreter's head [8]. Based on what has already been said by the speaker, the translator tries to predict what exactly will sound next.

If you work on consecutive translation, then you will have a few moments to improve the initial version a little - replace not very good words, change the passive voice to an active one, arrange the words in the sentence so that it sounds euphonious in the target language.

\section{RESEARCH ANALYSIS}

The specificity of the oral consecutive interpretation is determined by the general differences between interpretation and writing. Let us dwell on the most significant of them. First of all, one should speak about different types of perception (for oral translation auditory, for written - visual). In our opinion, the built-in procedure of oral translation is also indicative, which presupposes the active work of mental mechanisms and relatively permissible passivity of these mechanisms during the implementation of written translation. Both types of translation are inextricably linked with mental activity and are possible only in conditions of involvement of perception, attention, and memory. However, when performing a written translation, the translator's mind works in a less stressful mode, since his/her activity takes place in conditions of a sufficient amount of time both for reflection, consultation, rest, remembering, and for self-adjustment.

The features of OCI will be more obvious when compared with other types of interpreting activities, namely, sight translation and simultaneous translation. The main similarity of all types of interpretation is the temporal combination of the performed speech-thinking actions: listening and speaking with simultaneous translation; reading and speaking - when translating from sight; hearing and written fixation, reading the translation record and speaking - during the OCI [9].

According to many researchers, in order to achieve high results in teaching consecutive interpreting, it is 
necessary to train and develop various types of memory, which make it possible to correctly and effectively form the mechanisms serving the translator's activity, especially: a mechanism for assimilating the necessary knowledge based on the model of "adaptive control of the thinking process" - declarative, procedural and executive memory, mechanism for storing the received information, until it is issued in the target language working memory, the mechanism of long-term preservation of knowledge, skills and abilities - longterm memory, the mechanism of non-associative memorization - figurative memory, the mechanism of semantic grouping of a text during memorization is semantic memory [10].

Since the 90s of the twentieth century, the cognitive model of translation by D. Gilles, The Effort Models, has become widespread in foreign translation studies [11, p. 252]. The author presents translation as a cognitive process, consisting of a certain set of difficulties, in the resolution of which the translator needs to spend attention resources ("mental energy"). Each translation phase consists of a series of more or less resource-intensive operations. According to the concept of D. Gilles [11, p. 253], in consecutive translation at the first stage or phase, attention resources are involved, which are necessary for processing the information of original message, analyzing it, storing and retrieving background information from memory, resources spent on implementation of translation recording. At the same time, according to D. Gilles, writing down, the translator understands speech better in the process of its perception, however, excessive attention to the recording can lead to a deficit of attention resources [11, p. 256].

The results of the experiment conducted by D. Gilles led the author to a warning conclusion: what is not written down, the translator has a chance to remember, but what is not perceived will never be restored [11, p. 257]. In the second phase of translation, resources are involved that ensure the extraction of information from memory, the reading of the recording and generation of speech in the target language. Moreover, each of the process components is a "consumer" of attention resources, competes or interacts with each other: reading records helps to restore the original message in memory, while recalling the content of the original speech helps to generate it in the target language. The main conclusion that follows from the works of D. Gilles is the need to develop students-translators' skills to manage attention resources, namely, to correctly and efficiently distribute them, choose the most economical ways of fixing information, make decisions about the advisability of keeping a record or abandoning it focusing on speech perception [11, p. 259].

Cognitive theory has served as the methodological basis for a number of experimental studies on translation notation. In particular, K. Iliescu-Gheorghiu [12] evaluates the effectiveness of the translator's notes according to such criteria as the length of abbreviations, complexity / ease of writing symbols, designation of connectors, the scarcity / redundancy of the recording. J. Basten [13, p. 176], studying the texts of the recording, analyzes the ways of marking and conveying cohesion in consecutive translation, describes the typical difficulties of both professional and novice translators, which include simultaneity performing various actions and decrypting the records.

From the point of view of teaching consecutive translation, D. Robinson [14] believes that the translator equally needs all three types of memory to ensure the process of interpretation. Procedural memory is necessary for the subconscious processing of the text by the translator, and declarative memory is necessary for the conscious one. Procedural memory helps to translate quickly, and declarative memory is required in cases where problems that have arisen make fast translation impossible. Working memory in the process of consecutive interpretation is necessary to save the received information from the moment it is received until the moment it is issued in the target language.

The only repository of short-term memory has a limited volume and storage time, and the priority of information receipt is determined by the focus of attention at the moment. The storage time of information in operative memory is about 20 seconds, and its volume is 5-9 structural units of information - on average, 7 units (J. Miller's rule) [15, p. 176]. This volume is individual; it characterizes the natural memory of a person and tends to persist throughout life.

It can be increased in the case of using associative links, enlarging structural units, but not by increasing their number. J. Miller talks about the possibility of expanding the amount of memory through the construction of larger and larger segments containing more information than before. It can be 7 lexical units, represented by words, or maybe 7 semantic units, each of which is enclosed in several words, but contains one portion of information [15, p. 176]. At the same time, in the process of translation, the volume of the translation text decreases in comparison with the volume of the original text, that is, the translator applies speech compression.

Another characteristic of memory is fidelity, reflects a person's ability to accurately store, and most importantly, to reproduce information captured in memory. In the process of storing in memory, part of the information is lost, and part is distorted, and when reproducing this information, a person can make mistakes [16]. Therefore, the fidelity of reproduction, in our opinion, is a very significant characteristic of memory when teaching consecutive interpretation. 
Another important characteristic of memory is duration, reflects a person's ability to retain the necessary information for a certain time. This characteristic of operative memory is especially important for teaching consecutive translation, since the process of consecutive translation presupposes the presence of very long periods of "accumulation of information" by the translator, who is waiting for the end of the speaker's speech to complete the translation. Despite the "incompleteness" of consecutive translation, the main task of the translator, in our opinion, should be the complete fulfillment of communicative task. It is possible thanks to the actual division of the text. Actual division is the text structure at the logical-communicative level, in which each utterance consists of a topic (what is said about), a transitional element and a reme (what is said about the topic). The selection of topics and rem is a rather subjective thing and the translator can see in the text a logical-thematic structure that is different from the one that the author of this text sees in it, nevertheless the reliance on this structure serves as a "certain a guarantee of fulfillment of the main communicative task" [3, p. 107-108].

According to the nature of will participation in the process of memorization, involuntary and voluntary memory is distinguished. The effectiveness of voluntary memory depends on the goals of memorization and memorization techniques. At the same time, memorization techniques are: mechanical literal multiple repetition of the material; logical retelling, which includes logical comprehension of the material, systematization, highlighting the main logical components of information, retelling in your own words; figurative methods of memorization - translation of information into images, graphics, schemes, pictures (provided due to the work of figurative memory) $[3,8,9]$. As psychologists note, the use of mechanical memory is the most ineffective way of memorizing information, in contrast to logical (semantic) and figurative memorization techniques [4, p. 48]. Figurative memorization techniques effectively increase the capacity of operative memory.

This is a more persistent memory form, which is almost impossible to destroy, since it comes from the interests and professional knowledge of the person and is already inseparable from his/her personality. During teaching interpretation, the mechanisms' using of figurative memory, associative and non-associative memorization is a prerequisite for the development of memory for various types of precision information, the ability to "get used" to unexpected information and to memorize it in a general row, observing the requirement to preserve the sequence [3, p. 36]. The most effective way to develop a translator's memory is the semantic grouping of the text $[14$, p. 53]. Semantic grouping is the allocation of reference points that facilitate memorization. In addition, with oral consecutive translation, it is impossible to memorize all the words in a sentence, given the fact that all languages are redundant. The redundancy of language, especially in oral speech, is necessary for a person to be well understood, however, for the process of consecutive translation, it often becomes a stumbling block, as it overloads the operative memory and complicates the process of memorizing information.

Finally, it is necessary to consider the role of the longterm memory mechanism in the process of consecutive interpretation. First of all, it is necessary to define this type of memory. Long-term memory is a memory subsystem that provides a long-term preservation of temporary connections (skills) commensurate with life expectancy. It is believed that the amount of long-term memory is practically unlimited. Despite these valuable qualities of long-term storage, a person often does not get access to the knowledge stored there when the need arises. To reduce forgetting, it is necessary: understanding, comprehension of information, periodic repetition of information, creation of conditions for the student's interest in memorizing information [14, p. 73]. First of all, to describe the action of the mechanism of long-term memory in the process of consecutive translation, it should be mentioned that a number of authors directly point to the continuous circulation of information from working memory to long-term memory and vice versa. N.I. Zhinkin believes that lattices of phonemes and morphemes are formed in long-term memory, on which speech skills and abilities are based. In his opinion, the model that pops up in the mind is immediately filled with material from the lattice of morphemes, receiving in the speech the design corresponding to its situation [17, p. 28].

The interaction of operative memory and long-term memory is reduced to a comparison of information entering the working memory with the corresponding information stored in long-term memory and any changes in the content of long-term memory invariably entail corresponding changes in the work of the operative memory [17, p. 112-113].

\section{DISCUSSION OF SURVEY'S RESULTS}

A survey of masters, graduate students (practicing translators) majoring in "Philology" and "Translation Studies" was conducted, 30 students were interviewed. According to our survey, most students think that, from a psychological point of view, interpretation requires stable attention and memory, a clear and rapid response (Figure 1). The results of the study showed that in simultaneous interpretation, students have mainly the following difficulties: psychological discomfort (simultaneous listening and speaking) and mental stress caused by the rapid pace of the speaker's speech (Figure 2). According to the respondents, the 2 most important qualities that a professional real-time interpreter should have are speed of speech and accuracy of translation, mental and physical endurance (Figure 3 ). 
The results of the survey are illustrated in the following diagrams:

- $32 \%$ of respondents pointed that stable attention, memory and concentration $(22 \%$ of responders) are the most important qualities during simultaneous interpretation. It is necessary to have good memory, both short-term and operational and long-term (Figure 1).

- $25 \%$ of respondents noted that the interpreter must be physically resilient in order to withstand concentration and psychophysiological stress for a long time (Figure 2).

- $21 \%$ of respondents felt a mental stress caused by the rapid pace of the speaker's speech (Figure 2).

- $18 \%$ of interviewees wrote that interpreters could be able to understand acoustically correctly a foreign text performed by native speakers with different peculiarities of pronunciation, syntax and vocabulary without any problems (Figure 2).

- $35 \%$ of respondents answered that the interpreter has excellent diction and can convey information to listeners without loss and can speak for a long time without fatigue of the articular apparatus (Figure 3).

\section{CONCLUSION}

All of the above allows us to conclude that in order to carry out the consecutive translation process, the translator must be able to select from the speech stream those words that carry key information with the subsequent ability to compress the information received during translation, while preserving the content and all key elements of meaning. The allocation of key information or semantic support points occurs when using the attention mechanism. In the process of consecutive translation, the translator's attention is focused, step by step, first on the process of perceiving the original text, and then on the adequacy of his translation.

On the basis of the above memory mechanisms, the ability to predict, the ability to retain large chunks of information in memory, the ability to retain the information received until it is issued in the target language, the ability to associative memorization, the ability to semantic grouping of text when memorizing should be formed. In addition, the number of mental mechanisms that ensure the process of consecutive twoway translation include the mechanisms of perception of the original text, the design of the translation, synchronization of translation actions and the mechanism of switching - the transition from one language to another.

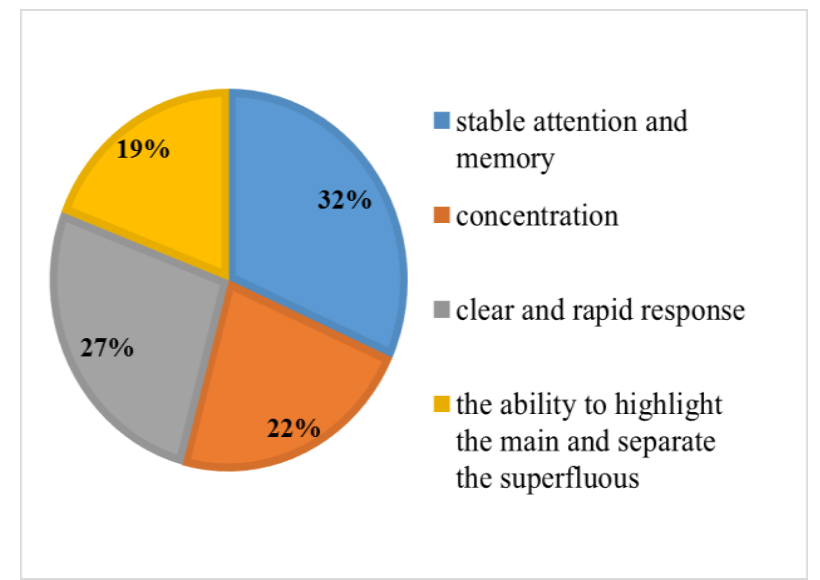

Figure 1. Interpretation as a complex receptivereproductive type of speech activity, from a psychological point of view requires: (choose 2 important positions, in your opinion).

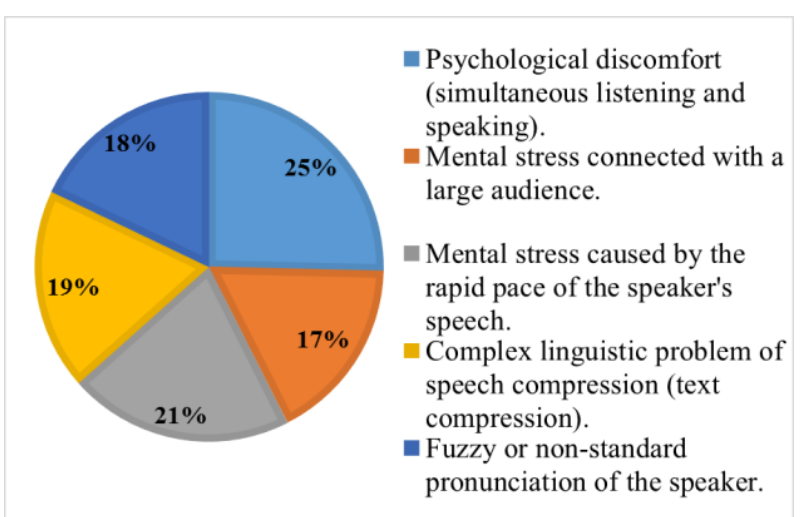

Figure 2. What difficulties do you have in simultaneous interpretation: (choose 2 positions).

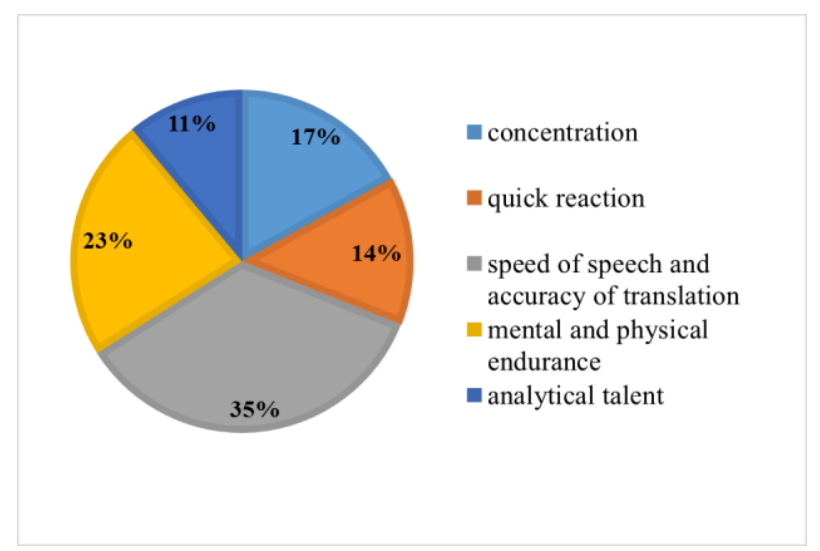

Figure 3. What qualities should a professional real-time interpreter have? Choose the 2 most important positions, in your opinion. 


\section{REFERENCES}

[1] Vikram Shweta and Sanjay K. Dwivedi (2018), "Ambiguity in Question Paper Translation", International Journal of Modern Education and Computer Science (IJMECS), vol. 10, no. 1, pp. 1323. DOI: $10.5815 /$ ijmecs.2018.01.02

[2] Kyrpychenko, Olena Pushchyna, Iryna Kichuk, Yaroslav Shevchenko, Nataliia Luchaninova, Olga and Koval, Viktor (2021), "Communicative Competence Development in Teaching Professional Discourse in Educational Establishments ", International Journal of Modern Education and Computer Science (IJMECS), vol. 13, Nn. 4, pp. 16-27. DOI: $10.5815 / \mathrm{ijmecs} .2021 .04 .02$

[3] Komisarov, V.N. (2011), Modern translation studies, Rev., R. Valent, Moscow, Rossia.

[4] Ermolovich, D.I. (2017), "Consecutive interpretation: the crisis of humanitarianism", Bulletin of Moscow University, vol. 9 (5), pp. 7994, available at: http://yermolovich.ru/index/0-221

[5] William, A. Kahn (1990), "Psychological Conditions of Personal Engagement and Disengagement at Work", The Academy of Management Journal, vol. 33, no. 4, pp. 692-724, available at: https://www.jstor.org/stable/256287

[6] Mavlevich, N.S. (2001), Translation - thinking in pictures", Russkij ZHurnal / Krug chteniya, available at: http://old.russ.ru/krug/20010425.html

[7] Linguistic code-switching: what it is and why it happens, available at: https://www.unitedlanguagegroup.com/blog/lingui stic-code-switching

[8] Yumeng, Lin Qianxi, Lv and Junying, Liang (2018), "Predicting Fluency With Language Proficiency, Working Memory, and Directionality in Simultaneous Interpreting”, Front. Psychol., 21 august. DOI: 10.3389/FPSYG.2018.01543

[9] I Wayan Suryasa, "The Comparison between Interpreting and Translation Process",
International Research Journal of Management IT and Social Sciences, vol. 3(1), p. 22, available at: https://www.researchgate.net/publication/3044546 68_The_Comparison_between_Interpreting_and_ Translation_Process

[10] Gile, D. (1992), "Les fautes de traduction: une analyse pédagogique", Meta, vol. 37 (2), pp. 251262, available at: https://www.erudit.org/en/ journals/meta/1900-v1-n1-meta336/002907ar.pdf

[11] Paul, F. M. J. (2016), "Synthetic consciousness: the distributed adaptive control perspective", Verschure, vol. 371, issue 1701, available at: https://doi.org/10.1098/rstb.2015.0448

[12] Iliescu Gheorghiu, C. (2001), "Introduccion a la interpretacion. La modalidad consecutive", Alicante: Universidad de Alicante, available at: https://www.euc.com/portades/9788497170840_L33_23.pdf

[13] Bastin, G. (2003), "Les marqueurs de cohérence en interprétation consecutive", The Interpreter Newsletter, vol. 2, pp. 175-187, available at: https://www.openstarts.units.it/bitstream/10077 12482/1/07.pdf

[14] Robinson, D. (2005), How to become a translator: an introduction to the theory and practice of translation, Kudits-Obraz, Moscow, Rosia, available at: https://library.tou.edu.kz/fulltext/buuk/b3141.p df

[15] Nelson, Cowan (2001), “The Magical Number 4 in Short-Term Memory: A Reconsideration of Mental Storage Capacity, Behavioral and Brain Sciences, Published online by Cambridge University Press, vol. 24 (1), 30 October, pp. 87-114. DOI:10.1017/S0140525X01003922

[16] Jitao, Xu Josep, Crego and Jean, Senellart (2020), "Boosting Neural Machine Translation with Similar Translations", available at: https://aclanthology.org/2020.acl-main.144.pdf

[17] Zhinkin, N.I. (1982), Speech as a conductor of information, Nauka, Moscow, Rossia. 